\title{
Phase I Clinical Trial of Everolimus Combined with Trimodality Therapy in Patients with Muscle-Invasive Bladder Cancer
}

Bassel G. Bachir ${ }^{\mathrm{a}}$, Luis Souhami ${ }^{\mathrm{b}}$, Jose João Mansure ${ }^{\mathrm{a}}$, Fabio Cury ${ }^{\mathrm{b}}$, Marie Vanhuyse ${ }^{\mathrm{c}}$, Fadi Brimo ${ }^{\mathrm{d}}$, Armen G. Aprikian ${ }^{\mathrm{a}}$, Simon Tanguay ${ }^{\mathrm{a}}$, Jeremy Sturgeon ${ }^{\mathrm{c}}$ and Wassim Kassouf ${ }^{\mathrm{a}, *}$

${ }^{a}$ Department of Urology, McGill University Health Center, Montreal, QC, Canada

${ }^{\mathrm{b}}$ Division of Oncology, Division of Radiation Oncology, McGill University Health Center, Montreal, QC, Canada

${ }^{\mathrm{c}}$ Department of Medical Oncology, McGill University Health Center, Montreal, QC, Canada

${ }^{\mathrm{d}}$ Department of Pathology, McGill University Health Center, Montreal, QC, Canada

\begin{abstract}
.
Background: Local control following trimodality therapy (TMT) for muscle-invasive bladder cancer (MIBC) requires further optimization.

Objective: Evaluating the biologic endpoint, feasibility, and toxicity of integrating everolimus to TMT in patients with MIBC. Methods: This was a phase I trial in patients with MIBC who were not surgical candidates or who refused cystectomy. Following maximal transurethral tumor resection, patients were treated by radiotherapy (50 Gy/20 fractions), gemcitabine ( $100 \mathrm{mg} / \mathrm{m} 2 /$ weekly) and escalating doses of everolimus $(2.5-5.0 \mathrm{mg} /$ day). Everolimus was given daily for one month prior to radiation, during treatment, and one month post-radiation. Toxicity assessment followed the Radiation Therapy Oncology Group Acute Radiation Morbidity Scoring Criteria. Biologic endpoint with downregulation of phospho-S6 (pS6) was assessed using immunohistochemistry. Local response was evaluated with imaging and bladder biopsy post-therapy.

Results: 10 patients were recruited; 8 males, 2 females. Median age was 78 years (range: 63-85). Four patients entered everolimus $2.5 \mathrm{mg}$ cohort. Six other patients entered everolimus $5.0 \mathrm{mg}$ cohort. Toxicities were encountered in 2 patients (Grade I), 6 patients (Grade II), 9 patients (Grade III) and 1 patient (Grade IV), with some experiencing more than one toxicity. Most Grade III and IV toxicities were encountered from everolimus alone prior to combination testing. Trial was terminated early due to toxicity. Interestingly, $6 / 10$ patients $(60 \%)$ achieved a complete response with negative post-treatment biopsies. Significant decrease of pS6 was demonstrated post-therapy $(p=0.03)$.

Conclusions: Although combining everolimus with TMT achieved a biological endpoint and complete response in a significant number of patients with $\mathrm{MIBC}$ and negative prognostic factors, it was associated with unacceptable increased toxicity.
\end{abstract}

Keywords: Everolimus, gemcitabine, intensity modulated radiotherapy, bladder cancer, dose hypofractionation

\footnotetext{
${ }^{*}$ Correspondence to: Wassim Kassouf, MD, FRCS(C), Department of Urology, McGill University Health Center, 1001 Decarie
} 


\section{INTRODUCTION}

A standard treatment for muscle-invasive bladder cancer (MIBC) remains radical cystectomy with pelvic lymph node dissection, with a $50 \%$ 5-year overall survival rate for cT2-4 tumors in contemporary large series [1-3].

However, a large number of patients referred for radical cystectomy either refuse surgery or are not surgical candidates due to associated competing comorbidities. Several centers have pioneered the bladder-preserving strategy as an alternative to radical cystectomy for MIBC over the last two decades [4-6]. It is particularly attractive as it attempts to spare the bladder, allowing for preservation of urinary and sexual functions, and avoiding the potentially significant morbidity of radical surgery, while maintaining an overall 5-year survival rate in wellselected patients similar to that achieved with radical cystectomy.

This strategy is multidisciplinary and includes maximal transurethral resection of bladder tumor (TURBT) followed by concurrent radiation therapy (RT) and chemotherapy (most often cisplatin-based). We recently published our results revealing significant antitumor activity through inhibition of the mTOR pathway with everolimus in bladder cancer models both in vivo and in vitro [7, 8]. Notably, we have shown that RT alone activated Akt/mTOR, a mechanism that may explain why certain bladder tumors are radioresistant. Ionizing radiation alters mRNA translation more profoundly than transcription. Furthermore, protein synthesis response to genotoxic stress caused by ionizing radiation seems to follow a biphasic response, with early, low dose exposure stimulating a rapid increase in protein synthesis via an increase of S6K and mTOR activities [9]. When everolimus was combined with RT, there was significant inhibition of proliferation and growth of bladder cancer cells both in vitro and in vivo.

On the basis of our experience along with an encouraging previous report of hypofractionated intensity modulated RT (IMRT) plus weekly gemcitabine that showed a response rate of $88 \%$ in frail patients with MIBC $[10,11]$ and based on the above and similar findings $[12,13]$, we initiated a phase I clinical trial of everolimus plus TMT to establish the toxicity of the combination and to potentially validate our hypothesis that this approach would improve local control of disease.

\section{MATERIALS AND METHODS}

\author{
Patient eligibility criteria and pretreatment \\ evaluation
}

This was a phase I prospective trial for patients with MIBC who were not surgical candidates because of associated significant medical comorbidities or who refused cystectomy. This trial received institutional review board approval at our center and patient consent was obtained. Eligible patients were 18 years old or older with a life expectancy greater than 3 months and ECOG performance status 2 or better. All had a histologic diagnosis of urothelial carcinoma of the bladder stage T2-T4a NOM0 (AJCC-TNM version 6) [14] based on TURBT and bimanual examination under anaesthesia. Focal differentiation was allowed, apart from small-cell histology. All patients were required to have a computed tomography (CT) scan of the abdomen/pelvis and chest X-ray within 6 weeks from the initiation of everolimus showing no evidence of metastatic disease. TURBT within 42 days (6 weeks) of the start of chemoradiation was performed.

Pretreatment evaluation included complete history and physical examination. All patients were deemed able to tolerate systemic chemotherapy combined with pelvic RT by the joint agreement of the participating radiation oncologist and medical oncologist.

Patients with prior systemic therapy for bladder cancer or metastatic disease were excluded as were patients who received prior gemcitabine, everolimuscontaining regimens or RT to the pelvis. Other exclusion criteria included pre-existing medical conditions precluding treatment, pregnancy or lactating mothers and patients unable to give informed consent.

\section{Treatment protocol}

The study was designed as a Phase I trial with fixed doses for RT and gemcitabine. The first cohort of patients would receive everolimus at a dose of $2.5 \mathrm{mg}$.

Cystoscopic evaluation included a maximal TURBT intended to remove all visible disease or suspicious lesions as well as a bimanual examination under anesthesia. Patients referred from outside institutions were re-resected by the participating urologist. Tissue sampling from the tumors was collected for translational studies. The primary end-point of the study was an assessment of toxicity from the combination of everolimus with TMT as well as biologic 


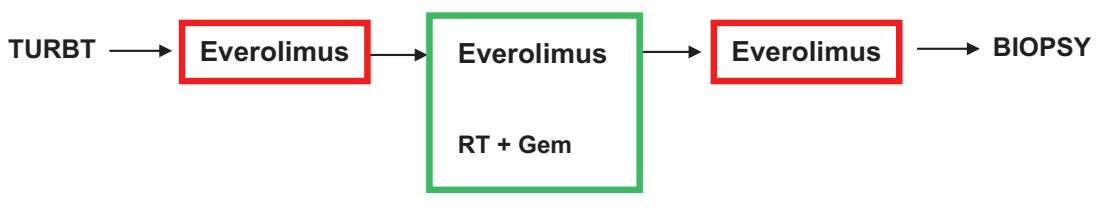

30 days

20 days

30 days

RT: $50 \mathrm{~Gy} / 20 \mathrm{fxs}$

Gem: $100 \mathrm{mg} / \mathrm{m}^{2}$

Fig. 1. Schematic diagram of treatment protocol.

endpoints (defined by downregulation of pS6) [7]. Local control rate as secondary endpoint was defined as a negative post-treatment biopsy.

RT was delivered daily, 5 days a week for a total of $50 \mathrm{~Gy}$ in 20 fractions. All patients were treated with IMRT. All patients underwent CT scan simulation and were required to have an empty bladder prior to simulation and before each RT treatment. IMRT was delivered with $6 \mathrm{MV}$ photon either by rapid arc or by 5 to 7 static fields. The clinical target volume (CTV) was the whole bladder (CTV tumor) and the pelvic lymph nodes including the obturator and external iliac nodes (CTV nodes). The CTV bladder was expanded 15 to $20 \mathrm{~mm}$ and the CTV node $7 \mathrm{~mm}$ to generate a planning target volume (PTV). Field-in-field technique was used to deliver $50 \mathrm{~Gy}$ in 20 fractions to the PTV tumor and $40 \mathrm{~Gy}$ in the same 20 fractions to the PTV nodes. Dose constraints for the organs at risk were as follows: rectum $\mathrm{V} 40 \leq 50 \%$, small bowel $\mathrm{V} 30 \leq 50 \%$, pelvic bone $\mathrm{V} 30 \leq 30 \%$ and femoral head Dmax $<40$ Gy.

All patients received concomitant weekly gemcitabine at a dose of $100 \mathrm{mg} / \mathrm{m}^{2}$, typically on days 1 , 8,15 and 22 of the RT. Everolimus was initially given at $2.5 \mathrm{mg} /$ day for 12 weeks, starting 1 month prior to TMT (Fig. 1) and continuing for an extra month after the concurrent chemoradiation was completed. Since the toxicity of everolimus alone has been already established, toxicity prior to TMT was of no direct interest in this trial but rather toxicity of the combined therapy. Three patients were to be treated at the protocol doses. Once these patients were accrued, the trial was placed on hold for 3 months until toxicity of the combination was assessed and felt to be safe. If one patient developed a grade 3 or higher toxicity due to the combined therapy, another 3 patients were to be studied. If another patient developed similar grade toxicity, the combination would be deemed too toxic. Once this was determined, everolimus was to be increased to $5 \mathrm{mg} /$ day. Again, 3 patients were to be treated at this level and observed. Once this dose was deemed tolerable, we would have considered this dose our standard everolimus dose and the study would continue thereafter as a Phase II. The gemcitabine and radiation dose was kept the same throughout the trial.

\section{Treatment evaluation}

The patients were assessed weekly by both medical and radiation oncology during active therapy. All follow-up assessments included physical examinations, and toxicity assessments. Gastrointestinal and genitourinary toxicities were graded according to the Radiation Therapy Oncology Group (RTOG) acute radiation morbidity scoring criteria, while the NCICTCAE (v. 3) was used for all others [15, 16]. Blood work was repeated weekly during treatment.

Enrolled patients (1st patient enrolled in July 2009; last patient January 2012) were followed by radiation oncology, medical oncology and urology in a multidisciplinary setting after completion of therapy every 3 months for the first 2 years, every 6 months for 2 years, then annually. Blood work was also repeated every 3 months for one year.

At 3 months post-treatment, patients underwent cystoscopic evaluation, and bimanual examination under general anesthesia with re-biopsy of previous tumor sites as well as any new suspicious areas. Complete response was defined as absence of residual cancer on post-treatment biopsy and no evidence of metastasis on imaging. Diagnostic cystoscopic evaluation without general anesthesia continued every 3 months for the first 2 years, every 6 months for 2 years, then annually. Urine cytology was collected at each subsequent cystoscopy visit. Imaging (chest Xray and $\mathrm{CT}$ abdomen/pelvis) was repeated at 3 months then every 6 months for 2 years. 
Analysis of expression of downregulation of phospho-S6 (pS6), a member of the Akt/mTOR signalling pathway, was performed using immunohistochemistry on the pre- and 1 month post-therapy biopsies in 3 patients with residual disease. Briefly, formalin-fixed paraffin-embedded sections were deparaffinized in xylene and hydrated with changes of $100 \%$ ethanol, followed by $95 \%$ ethanol and distilled water. Antigen-retrieval was performed by heating the slides with sodium citrate buffer $(10 \mathrm{mM}$ Sodium Citrate, $\mathrm{pH}$ 6.0). Peroxidase blocking was done in $3 \%$ $\mathrm{H}_{2} \mathrm{O}_{2}$ /PBS solution for 10 min followed by washes in PBS-Tween. To assess the levels of pS6 expression, sections were incubated overnight at $4^{\circ} \mathrm{C}$, with primary specific antibodies against pS6 (Cell Signaling Technology, New England MA; 1:200 dilution). HRP-conjugated goat polyclonal anti-rabbit IgG secondary antibody was added and incubated at room temperature for one hour. After washing, reactions were revealed by incubating sections with 3,3'Diaminobenzidine (DAB) substrate (Sigma-Aldrich, Canada) according to manufacturer's instructions and counterstained with hematoxylin. Slides were viewed by light microscopy under a Leica Diaplan inverted microscope (Leica Inc.) by a single pathologist blinded to treatment status. Analysis of staining was based on an average of 5 foci, at $40 \mathrm{X}$ magnification, and a completed score was calculated by summing the products of the percentage cells at a given staining intensity (0-100) and the staining intensity ( 0 for negative, 1 for low and 2 for moderate and 3 for high staining).

\section{RESULTS}

A total of 10 patients entered the study with median follow-up of 36 months (range 9 to 69). Eight males and 2 females with a median age of 78 years (range: 63-85). Overall, $40 \%$ of patients had $\geq \mathrm{cT} 3 \mathrm{~b}$ disease and $50 \%$ of patients with cT2 had concomitant CIS. Four patients entered the everolimus $2.5 \mathrm{mg}$ cohort. When this dose was deemed safe, six patients then entered the everolimus $5.0 \mathrm{mg}$ cohort. Details of both groups of patients are found in Table 1. All patients completed the required IMRT sessions. Six patients $(60 \%)$ completed 4 cycles of gemcitabine.

\section{Everolimus $2.5 \mathrm{mg}$ cohort}

A total of 4 patients entered this cohort ( 3 males, one female). Toxicity of the combination could not be

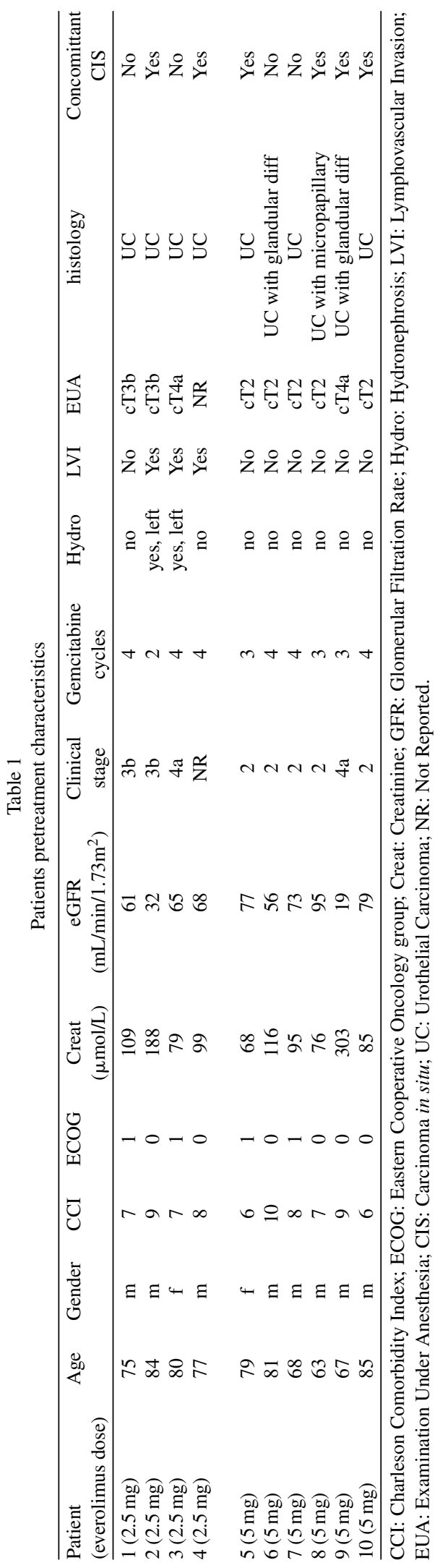


Table 2

Details of toxicity

\begin{tabular}{|c|c|c|c|c|c|}
\hline \multirow{2}{*}{$\begin{array}{l}\text { Everolimus dose } \\
\text { Grade }\end{array}$} & \multicolumn{3}{|c|}{$2.5 \mathrm{mg}$} & \multicolumn{2}{|c|}{$5.0 \mathrm{mg}$} \\
\hline & G1 & G2 & G3 G4 G1 & G2 & $\overline{G 3}$ G4 \\
\hline \multicolumn{6}{|l|}{ Hematologic toxicity } \\
\hline Not specified & & & 1 & & 3 \\
\hline Anemia & & 1 & & & \\
\hline Lymphopenia & & 2 & & & 1 \\
\hline High INR & & & & & 1 \\
\hline \multicolumn{6}{|l|}{ Pancytopenia } \\
\hline Neutropenia & & & & & 1 \\
\hline \multicolumn{6}{|c|}{ Non-hematologic toxicity } \\
\hline Diarrhea & 1 & 1 & & 1 & 1 \\
\hline Urinary frequency & & 2 & & & \\
\hline $\begin{array}{l}\text { Hepatic } \\
\text { (High LFT's) }\end{array}$ & & & 1 & & 3 \\
\hline Skin rash & 1 & & 1 & & 1 \\
\hline Sigmoiditis & & & & 1 & \\
\hline Fatigue & & & & & 1 \\
\hline Cystitis & & & & & 1 \\
\hline $\begin{array}{l}\text { Congestive } \\
\text { heart failure }\end{array}$ & & & & 1 & \\
\hline Proctitis & & & & 1 & 1 \\
\hline Chronic renal failure & & & & & 1 \\
\hline Low albumin & & & & & 1 \\
\hline
\end{tabular}

assessed in one patient as he never ended up receiving the combination therapy due to altered liver function tests (grade 3 ) following initiation of everolimus alone, prior to exposure to the combined therapy. Ten months following treatment completion, he died of pneumonia that was felt to be unrelated to his disease or treatment. Of interest, this patient's bladder biopsy after completion of his therapy showed no residual cancer. Details of toxicity are shown in Table 2. Four patients completed the $2.5 \mathrm{mg}$ cohort.

\section{Everolimus $5.0 \mathrm{mg}$ cohort}

A total of 6 patients entered this cohort (5 males, one female). Details of toxicity are shown in Table 2. All of the patients had more than one type of toxicity and/or grade. Most Grade 3 and 4 toxicities were encountered from everolimus alone prior to combination testing. Additionally, although all of the previously reported toxicity resolved, the study was terminated early due to increased toxicity at this dose level in the combined therapy (Table 2).

\section{Response}

Everolimus induced a biologic response with significant decrease of pS6 as was demonstrated on post therapy biopsies $(p<0.03)$ (Fig. 2). Six out of the ten patients $(60 \%)$ achieved a complete clinical response with negative post-treatment biopsies, with four of the six patients from the everolimus $5.0 \mathrm{mg}$ cohort and two from the $2.5 \mathrm{mg}$ cohort. All patients who did achieve a complete response remain alive and free of recurrences in their bladder. Of those who did not achieve a complete response, 3 died of disease and 1 remained alive without evidence of disease following salvage cystectomy.

\section{DISCUSSION}

Although combining everolimus with TMT showed promising results with an encouraging number of post treatment negative biopsies and a significant downregulation of $\mathrm{pS6}$, the trial was terminated due to unacceptable acute toxicity of everolimus combined with TMT.

The ability to eradicate malignant tumors while preserving organs and their function has been changing oncological practice over the years. A multimodality approach has also been employed in the treatment of cancers of the head and neck, anus, rectum, and soft tissue sarcomas as well as bladder cancer, providing acceptable disease control while maintaining good organ function and quality of life.

Two phase III trials have demonstrated the superiority of combining radiotherapy with other treatments over radiotherapy alone in the treatment of muscleinvasive bladder cancer. James et al. demonstrated the superiority of combined chemoradiation in the treatment of MIBC, with the primary synergistic effect being the radiosensitization offered by using combination chemotherapy of fluorouracil and mitomycin C [17]. Alternatively, the BCON study combined radiotherapy with agents that induce tumor hypoxia, including nicotinamide and carbogen [18]. Both of these studies show that agents that act by enhancing the effect of radiotherapy do in fact have a beneficial effect on outcome in patients treated with organ sparing therapies for MIBC.

Gemcitabine has also been found to be a potent radiosensitizing agent in phase I and II clinical trials with an acceptable safety profile [10, 19-23]. Since an activated Akt/mTOR pathway has also been implicated in radioresistance and disease recurrence $[24,25]$, the hypothesis of our research team was that combining everolimus with TMT may increase radiosensitization in the clinical setting, similar to what we have found in our preclinical bladder cancer models. Although the complete response following TMT (CR 60\%) was lower than reported in the literature, it is noteworthy to highlight that patient tumor 

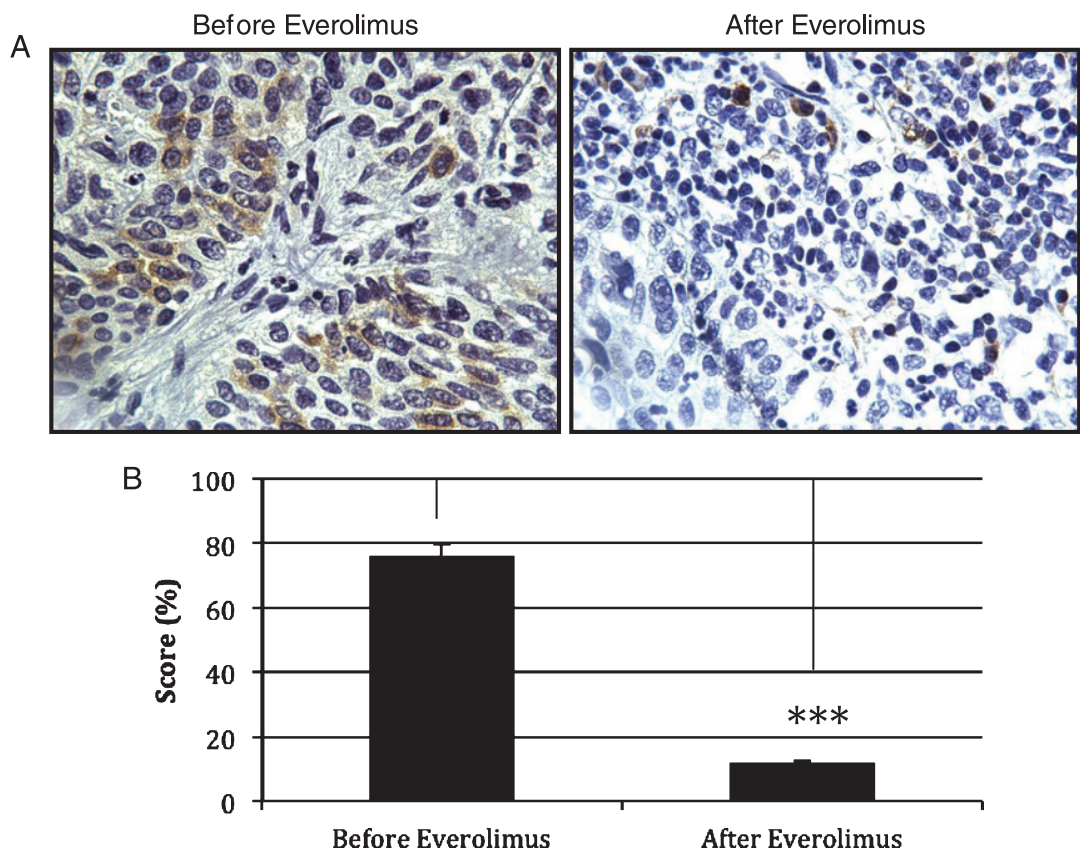

Fig. 2. Expression of pS6 as measured of mTOR activity. (A) Immunohistochemistry was used to detect the levels of pS6 in paraffin-embedded tissues of patients treated with Everolimus. (B) Quantification of the immunohistochemistry data in 3 patients with residual disease $(n=3)$ revealed a significant decrease in pS6 expression as observed in tumors treated with Everolimus $(p=0.021)$. Treatment of Everolimus started 4 weeks before the combined gemcitabine/radiotherapy treatment. Everolimus was continued during the chemoradiation regimen and for one extra month after the concurrent chemoradiation regimen has been completed.

profile is not typical of those reported in the literature; the majority of the patients had negative prognostic factors of response (high volume disease with $40 \%$ of patients being $\geq$ cT $3 \mathrm{~b}$ ) or concomitant CIS in $50 \%$ of those with cT2 disease). However, the combination of everolimus, gemcitabine and IMRT was associated with increased acute toxicity. It is likely, in part, that the toxicity profiles of gemcitabine and everolimus overlapped, leading to the findings noted in this study. Other studies have also recently demonstrated everolimus to be too toxic when combined with cisplatin-based combination chemotherapy used for advanced urothelial carcinoma, even without the addition of radiotherapy [26]. This problem may have been potentially avoided either by decreasing drug concentrations or alternatively switching to a combination of drugs and treatments that do not have an additive/synergistic toxic effect. In the case of everolimus, should we have studied the $2.5 \mathrm{mg}$ dose further as this dose seems to have been better tolerated by our patients than the $5.0 \mathrm{mg}$ dose? In the case of gemcitabine, the dose used in this study is already one tenth of the dose that is commonly used in the neoadjuvant setting, with the benefit of this drug manifested mostly in its radiosensitizing effect rather than a cytotoxic effect [10]. Although cisplatin is commonly used in patients treated with TMT, our center have routinely used gemcitabine in the elderly patients, which primarily comprised the current study's patient population. With our hypofractionated daily dose approach of IMRT, we were able to reduce scatter to normal organs, resulting in less radiation toxicity than what has been observed in other trials using twice daily fractionation or conventional RT [27-30]. Two reports by Hsieh et al. and van Rooijen et al. where patients were treated with RT for MIBC demonstrated a superior normal organ sparing ability through the adoption of IMRT [31, 32]. In our published experience, the use of TMT with IMRT and weekly gemcitabine did not lead to an increased toxicity rate [11]. This is again of utmost importance particularly in the elderly population that we are treating. Nevertheless, despite all of this and our best attempts at reducing the toxicity of our treatment, we still found that the combination used was too toxic. There are some limitations to this study including the small number of patients recruited, incomplete target accrual (a phase 2 was never initiated), as well as the possibility of patient selection bias. Furthermore, since this was a single arm study, we could not assess downregulation of $\mathrm{pS} 6$ in patients treated with TMT who did not receive everolimus. 


\section{CONCLUSION}

Although the combination of everolimus with gemcitabine and IMRT achieved a biological endpoint and a complete local response in a significant number of patients with MIBC and negative prognostic factors, it was associated with increased acute toxicity.

\section{ACKNOWLEDGMENTS}

Dr Wassim Kassouf is a recipient of a Research Scholar Award from the FRSQ.

\section{CONFLICT OF INTEREST}

The authors have no conflict of interest to report.

\section{REFERENCES}

[1] Rodel C, Weiss C, Sauer R. Organ preservation by combined modality treatment in bladder cancer: The European perspective. Semin Radiat Oncol 2005;15(1):28-35.

[2] Dalbagni G, Genega E, Hashibe M, Zhang ZF, Russo P, Herr $\mathrm{H}$, et al. Cystectomy for bladder cancer: A contemporary series. The Journal of Urology 2001;165(4):1111-6.

[3] Stein JP, Lieskovsky G, Cote R, Groshen S, Feng AC, Boyd $\mathrm{S}$, et al. Radical cystectomy in the treatment of invasive bladder cancer: Long-term results in 1,054 patients. Journal of Clinical Oncology: Official Journal of the American Society of Clinical Oncology 2001;19(3):666-75.

[4] Housset M, Maulard C, Chretien Y, Dufour B, Delanian $\mathrm{S}$, Huart $\mathrm{J}$, et al. Combined radiation and chemotherapy for invasive transitional-cell carcinoma of the bladder: A prospective study. Journal of Clinical Oncology: Official Journal of the American Society of Clinical Oncology 1993;11(11):2150-7.

[5] Sauer R, Dunst J, Altendorf-Hofmann A, Fischer H, Bornhof C, Schrott KM. Radiotherapy with and without cisplatin in bladder cancer. Int J Radiat Oncol Biol Phys 1990;19(3):687-91.

[6] Shipley WU, Kaufman DS, Tester WJ, Pilepich MV, Sandler HM. Overview of bladder cancer trials in the Radiation Therapy Oncology Group. Cancer. 2003;97(8 Suppl): 2115-9.

[7] Mansure JJ, Nassim R, Chevalier S, Rocha J, Scarlata E, Kassouf W. Inhibition of mammalian target of rapamycin as a therapeutic strategy in the management of bladder cancer. Cancer Biol Ther 2009;8(24):2339-47.

[8] Nassim R, Mansure JJ, Chevalier S, Cury F, Kassouf W. Combining mTOR inhibition with radiation improves antitumor activity in bladder cancer cells in vitro and in vivo: A novel strategy for treatment. PLoS One 2013;8(6): e65257.

[9] Braunstein S, Badura ML, Xi Q, Formenti SC, Schneider RJ. Regulation of protein synthesis by ionizing radiation. Mol Cell Biol 2009;29(21):5645-56.

[10] Choudhury A, Swindell R, Logue JP, Elliott PA, Livsey JE, Wise M, et al. Phase II study of conformal hypofractionated radiotherapy with concurrent gemcitabine in muscle-invasive bladder cancer. Journal of Clinical Oncology: Official Journal of the American Society of Clinical Oncology 2011;29(6):733-8.

[11] Turgeon GA, Souhami L, Cury FL, Faria SL, Duclos M, Sturgeon J, et al. Hypofractionated intensity modulated radiation therapy in combined modality treatment for bladder preservation in elderly patients with invasive bladder cancer. Int J Radiat Oncol Biol Phys 2014;88(2):326-31.

[12] Houédé N, Pourquier P. Targeting the genetic alterations of the PI3K-AKT-mTOR pathway: Its potential use in the treatment of bladder cancers. Pharmacol Ther 2015; 145(Journal Article):1-18.

[13] Pinto-Leite R, Arantes-Rodrigues R, Sousa N, Oliveira PA, Santos L. mTOR inhibitors in urinary bladder cancer. Tumour Biol 2016;37(9):11541-51.

[14] Greene FL, American Joint Committee on Cancer., American Cancer Society. AJCC cancer staging manual. 6th ed. New York: Springer-Verlag; 2002. xiv, 421.

[15] http://www.rtog.org

[16] ctep.cancer.gov

[17] James ND, Hussain SA, Hall E, Jenkins P, Tremlett J, Rawlings $\mathrm{C}$, et al. Radiotherapy with or without chemotherapy in muscle-invasive bladder cancer. The New England Journal of Medicine 2012;366(16):1477-88.

[18] Hoskin PJ, Rojas AM, Bentzen SM, Saunders MI. Radiotherapy with concurrent carbogen and nicotinamide in bladder carcinoma. Journal of Clinical Oncology: Official Journal of the American Society of Clinical Oncology. 2010;28(33):4912-8.

[19] Fields MT, Eisbruch A, Normolle D, Orfali A, Davis MA, $\mathrm{Pu}$ AT, et al. Radiosensitization produced in vivo by oncevs. twice-weekly 2'2'-difluoro-2'-deoxycytidine (gemcitabine). Int J Radiat Oncol Biol Phys 2000;47(3):785-91.

[20] Lawrence TS, Chang EY, Hahn TM, Hertel LW, Shewach DS. Radiosensitization of pancreatic cancer cells by $2^{\prime}, 2^{\prime}-$ difluoro-2'-deoxycytidine. Int J Radiat Oncol Biol Phys 1996;34(4):867-72.

[21] Mason KA, Milas L, Hunter NR, Elshaikh M, Buchmiller L, Kishi K, et al. Maximizing therapeutic gain with gemcitabine and fractionated radiation. Int J Radiat Oncol Biol Phys. 1999;44(5):1125-35.

[22] Pacini S, Milano F, Pinzani P, Pazzagli M, Gulisano M, Ruggiero M, et al. Effects of gemcitabine in normal and transformed human lung cell cultures: Cytotoxicity and increase in radiation sensitivity. Tumori 1999;85(6): 503-7.

[23] Shewach DS, Lawrence TS. Radiosensitization of human solid tumor cell lines with gemcitabine. Semin Oncol 1996;23(5 Suppl 10):65-71.

[24] Zhan M, Han ZC. Phosphatidylinositide 3-kinase/AKT in radiation responses. Histol Histopathol 2004;19(3):915-23.

[25] Kim TJ, Lee JW, Song SY, Choi JJ, Choi CH, Kim BG, et al. Increased expression of pAKT is associated with radiation resistance in cervical cancer. Br J Cancer 2006;94(11):167882.

[26] Abida W, Milowsky MI, Ostrovnaya I, Gerst SR, Rosenberg JE, Voss MH, et al. Phase I Study of Everolimus in Combination with Gemcitabine and Split-Dose Cisplatin in Advanced Urothelial Carcinoma. Bladder Cancer 2016;2(1):111-7.

[27] Tester W, Porter A, Asbell S, Coughlin C, Heaney J, Krall J, et al. Combined modality program with possible organ preservation for invasive bladder carcinoma: Results of RTOG protocol 85-12. Int J Radiat Oncol Biol Phys 1993;25(5):783-90. 
[28] Shipley WU, Winter KA, Kaufman DS, Lee WR, Heney NM, Tester WR, et al. Phase III trial of neoadjuvant chemotherapy in patients with invasive bladder cancer treated with selective bladder preservation by combined radiation therapy and chemotherapy: Initial results of Radiation Therapy Oncology Group 89-03. Journal of Clinical Oncology: Official Journal of the American Society of Clinical Oncology 1998;16(11):3576-83.

[29] Hagan MP, Winter KA, Kaufman DS, Wajsman Z, Zietman AL, Heney NM, et al. RTOG 97-06: Initial report of a phase I-II trial of selective bladder conservation using TURBT, twice-daily accelerated irradiation sensitized with cisplatin, and adjuvant MCV combination chemotherapy. Int J Radiat Oncol Biol Phys 2003;57(3):665-72.
[30] Rodel C, Grabenbauer GG, Kuhn R, Papadopoulos T, Dunst J, Meyer M, et al. Combined-modality treatment and selective organ preservation in invasive bladder cancer: Long-term results. Journal of Clinical Oncology: Official Journal of the American Society of Clinical Oncology 2002;20(14):3061-71.

[31] Hsieh CH, Chung SD, Chan PH, Lai SK, Chang HC, Hsiao $\mathrm{CH}$, et al. Intensity modulated radiotherapy for elderly bladder cancer patients. Radiat Oncol 2011;6:75.

[32] van Rooijen DC, van de Kamer JB, Hulshof MC, Koning CC, Bel A. Improving bladder cancer treatment with radiotherapy using separate intensity modulated radiotherapy plans for boost and elective fields. J Med Imaging Radiat Oncol 2010;54(3):256-63. 